\title{
A look on trauma code activation in a major trauma centre in UAE: a descriptive study
}

\author{
Mustafa Mahmood Eid*, Maythem Al-Kaisy \\ Emergency Department, Al Ain Hospital, Al Ain, United Arab Emirates
}

\begin{abstract}
Received: 4 April 2020
Accepted: 17 May 2020

Published online: 6 June 2020

*Corresponding author: Mustafa Mahmood Eid, Shakboot Bin Sultan Street, Al Ain, P.O. Box 1006, United

Arab Emirates.

Email:

Dr.mustafa191982@gmail.com

Competing interests: None.

Funding information: None.

Citation: Eid MM, Al-Kaisy M. A look on trauma code activation in a majo trauma centre in UAE: a descriptive study. Journal of Emergency Practice and Trauma 2020; 6(2): 82-86. doi:

10.34172/jept.2020.16.
\end{abstract}

\begin{abstract}
Objective: Trauma is considered to be a major cause of morbidity and mortality all over the world. This descriptive study has an emphasize on the epidemiology, mechanism and patterns of trauma, with a consideration of why trauma code was activated, and the imaging results in regard to the severity of the trauma.

Methods: A descriptive study was conducted in Al Ain Hospital over the year of 2017. Totally 886 patients were included who presented with a trauma and considered dangerous according to hospital guidelines. They underwent a full body trauma CT, and were admitted to the hospital. We looked at the mechanisms, patterns, time of the day and radiological findings. Injury severity score (ISS) and Revised trauma score (RTS) were calculated, and the cause of trauma code activation was evaluated.

Results: The study showed that; positive imaging findings were found in 364 (41\%) of patients while $522(59 \%)$ had normal radiological tests. The principal mechanism of injury was motor vehicle accident $(54.4 \%)$ followed by falls $(21.4 \%)$ and pedestrian accidents (10.2\%). Overall, $69.75 \%$ (618 patients) were admitted to the hospital, 22 patients (2.5\%) needed immediate interventions and 36 patients (4\%) needed ICU admission. Only 2 patients $(0.25 \%)$ died in the emergency room.

Conclusion: The hospital policy in activating a trauma code should be revised, with more care being paid to the mechanisms of injury and the condition of the patient, without missing any injury that could harm the patient. So, the emergency physician should be better prepared to do a detailed physical examination and weigh the risk of radiation against missing a dangerous injury.

Keywords: Trauma code, Trauma CT, Radiological findings, Hospital policy
\end{abstract}

\section{Introduction}

Trauma is a leading cause of mortality. Globally, more than 45 million people sustain moderate to severe disability each year due to trauma (1) where road traffic injuries are the major cause of death between the ages of 18 and 29. In the United States, trauma continues to be the main cause of death in young adults and accounts for 10 percent of all deaths among men and women (2). Trauma is considered to be the dominant cause of mortality all over the world in patients less than 44 years of age and the $4^{\text {th }}$ reason for death in all high-income countries (3). Survivors usually undergo a long-term process of rehabilitation and complex reintegration into their previous normal lives, making it a principal socioeconomic load on both the patients and the community in general, with an exponential increase in health costs (4).

Certain mechanisms are prone to specific injuries. Besides, certain high-risk mechanisms, including severe motor vehicle accidents, pedestrians struck by automobiles, motorcycle accidents, and falls greater than 6 meters, have been correlated with greater morbidity and mortality (57). Al-Ain is a growing city of population reaching about 767000 people according to the latest data, with two major governmental hospitals. Al-Ain hospital is a major trauma center receiving the majority of trauma patients with the availability of all specialties, imaging and operating rooms. The burden of unintentional injuries is growing and in order to estimate the number of patients admitted to the hospital, a trauma registry book was developed in AlAin Hospital and it is updated on a day to day basis. The registry book includes all kinds of trauma from simple cases to severe ones. The objective of our study was to study the descriptive epidemiology, mechanisms, and patterns of major trauma presenting to Al-Ain Hospital emergency department while focusing on inspecting, evaluating, and commenting on the images taken by following the hospital guidelines. 


\section{Methods}

This cross-sectional study was undertaken in the emergency department of Al-Ain hospital. The data were evaluated from the hospital trauma registry from 1 January 2017 to 31 December 2017. The required data were extracted from the hospital trauma registry. Each patient's profile was evaluated separately. We evaluated the mechanism, pattern, time of the day, age, sex, CT findings, Revised Trauma Score (RTS) [which is a physiologic scoring system that quantifies the severity of trauma injuries based on Glasgow Coma Scale (GCS), blood pressure, and respiratory rate], and Injury Severity Score (ISS) [which is a medical score to assess trauma severity and standardizes the severity of traumatic injury based on the worst injuries from 6 body systems].

All patients presenting with a major trauma according to our hospital guidelines (Figure 1) were included in the study. We excluded patients who refused to do the whole-body trauma computed tomography (CT) scan from our study. All included patients underwent trauma code activation where a whole-body trauma CT (including head, cervical spine, chest, abdomen/pelvis, thoracic and lumbar spine) was conducted and patients were admitted at least one day for observation. In this regard, we evaluated our hospital guidelines for initiating a trauma code by the emergency medicine physician and did a trauma CT with the findings in CT scans. Trauma registry data were collected after obtaining approval from hospital administration (No. 0936). We did not seek for the approval of patients as no individual information was available.

We divided the patients into 4 groups according to ISS score, and evaluated each group for the kinds of trauma and disposition. We then did the same calculation but in regard to the RTS. Diurnal variations of trauma also were looked at, in which the day was divided into 3 periods according to our shift timings, morning from 7 AM to 3 $\mathrm{PM}$, evening from $3 \mathrm{PM}$ to $11 \mathrm{PM}$ and night from 11 PMto 7 AM.
We also evaluated the trauma code activation protocol in our hospital, in which trauma code was activated and the whole-body CT trauma was done. We matched the protocol with our CT findings. All patients' data were entered by using computerized statistical software; Statistical Package for Social Science (SPSS) version 21. Kolmogorov-Smirnov analysis verified the normality of data set. Multiple contingency table analysis was conducted and appropriate statistical tests were performed. In all statistical analyses, level of significance was set at $P$ value $<$ 0.05 . The obtained results are presented as tables and/or graphs.

\section{Results}

Over a period of one year, 886 patients were included in the study, of which 718 were males and 168 were females, with a male to female ratio of $4.3: 1$. The youngest patient was one year old, while the oldest patient was 68 years old, with an average of 32.6 (Figure 2).

Positive imaging findings were found in 364 patients (41\%), while 522 patients (59\%) had normal radiological tests. The principal mechanism of injury was motor vehicle accident $(54.4 \%)$ followed by falls $(21.4 \%)$ and pedestrian accidents (10.2\%) (Table 1). The most frequently injured

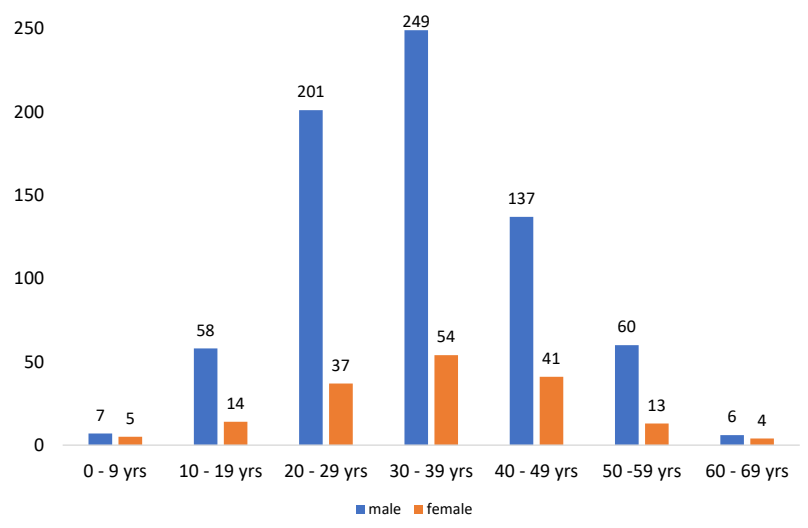

Figure 2. Age and gender distribution of trauma patients.

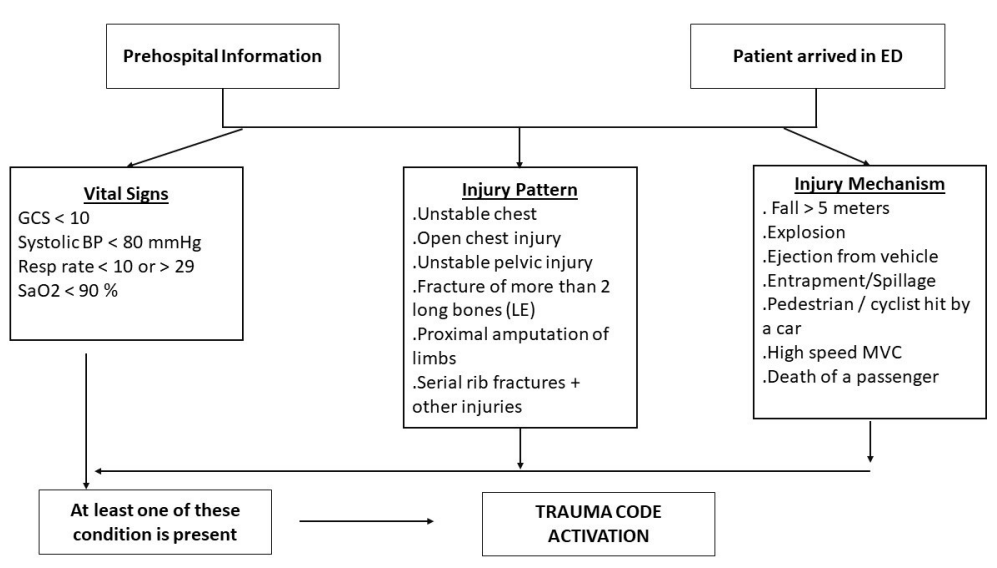

Figure 1. Trauma code activation protocol in Al-Ain Hospital. 
Table 1. Mechanisms of trauma in relation to imaging findings

\begin{tabular}{lccc}
\hline Type of Trauma & $\begin{array}{c}\text { Positive CT } \\
\text { Findings }\end{array}$ & $\begin{array}{c}\text { Negative } \\
\text { CT Findings }\end{array}$ & $\begin{array}{c}\text { Total } \\
\text { Number }\end{array}$ \\
\hline Motor vehicle & 176 & 306 & $482(54.4 \%)$ \\
\hline Pedestrian V/S Car & 50 & 40 & $90(10.2 \%)$ \\
Falls & 82 & 108 & $190(21.4 \%)$ \\
\hline Bicycle and motor bike & 34 & 36 & $70(7.9 \%)$ \\
\hline Assault & 8 & 14 & $22(2.5 \%)$ \\
Burn & 2 & 8 & $10(1.1 \%)$ \\
\hline Others* & 12 & 10 & $22(2.5 \%)$ \\
\hline Total & $364(41 \%)$ & $522(59 \%)$ & 886 \\
\hline
\end{tabular}

*Includes strangulation, stab wound, fall of heavy objects, injuries by camel and explosion of cooking gas tanks.

$P$ value: 0.0069, Chi-square test.

Table 2. Percentages of the body parts injured

\begin{tabular}{lcc}
\hline Body Part Injured & Number of Patients & Percent \\
\hline Head and neck & 130 & 14.7 \\
Chest & 124 & 14 \\
Abdomen & 30 & 3.4 \\
Pelvis & 70 & 8 \\
Spine & 108 & 12 \\
\hline
\end{tabular}

body parts were head and neck (14.7\%), chest (14\%), as well as spinal injuries (12\%) (Table 2). It is important to mention that some patients had injuries in more than one part of their bodies, thus the total number was higher than

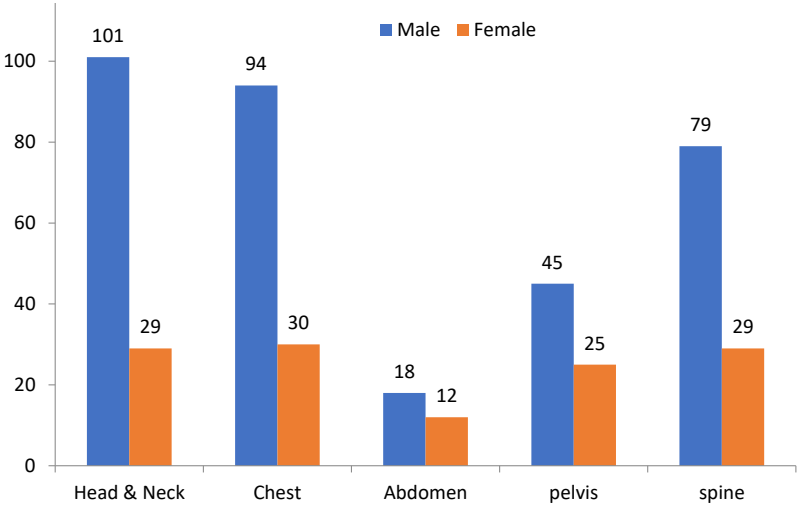

Figure 3. Percentages of the body parts injured with male and female ratio

the total positive CT findings. The male to female ratio according to the injured parts is displayed in Figure 3.

Severity of injury was defined ISS $>24$ and accounted for $8.1 \%$, while according to RTS it was defined by a score of $\leq 5$ and accounted for only $1.8 \%$ (see Tables 3 and 4 ). The majority of trauma codes occurred in the evening shifts (51.9\%), followed by morning shifts (30.4\%) (Table 5).

Overall, 618 patients $(69.75 \%)$ were admitted to the hospital, and only 22 patients $(2.5 \%)$ needed immediate interventions, while 36 patients (4\%) needed ICU admission, and only 2 patients $(0.05 \%)$ died in the emergency room. Sixty-eight patients $(7.7 \%)$ refused to stay at the hospital and preferred to leave against medical advice (LAMA) due to different reasons like feeling well, financial issues or personal reasons.

Table 3. Trauma mechanisms in relation to ISS

\begin{tabular}{|c|c|c|c|c|c|c|}
\hline Variable & ISS $<12$ & ISS 12-15 & ISS 16-24 & ISS > 24 & Total & P value \\
\hline Patients & $724(81.7 \%)$ & $36(4.1 \%)$ & $54(6.1 \%)$ & $72(8.1 \%)$ & 886 & \\
\hline \multicolumn{7}{|l|}{ Age } \\
\hline Average (y) & $30.3 \pm 13.4$ & $29.5 \pm 13.76$ & $34.6 \pm 7.45$ & $36.6 \pm 17.56$ & & \\
\hline \multicolumn{7}{|l|}{ Gender } \\
\hline Male & $576(65 \%)$ & 34 (3.83\%) & $46(5.1 \%)$ & $62(6.99 \%)$ & $718(87.8 \%)$ & \\
\hline Female & $148(16.7 \%)$ & $2(0.22 \%)$ & $8(0.9 \%)$ & $10(1.12 \%)$ & $168(18.96 \%)$ & \\
\hline \multicolumn{7}{|l|}{ Trauma type } \\
\hline Motor vehicle collision & $408(46 \%)$ & $12(1.35 \%)$ & $24(2.7 \%)$ & $38(4.3 \%)$ & $482(54.4 \%)$ & 0.033 \\
\hline Pedestrian & $68(7.7 \%)$ & $4(0.45 \%)$ & $8(0.9 \%)$ & $10(1.1 \%)$ & $90(10.1 \%)$ & \\
\hline Fall & $156(17.6 \%)$ & $14(1.6 \%)$ & $12(1.35 \%)$ & $8(0.9 \%)$ & $190(21.4 \%)$ & \\
\hline Bicycle/motor bike & $56(6.3 \%)$ & $2(0.2 \%)$ & $6(0.7 \%)$ & $6(0.7 \%)$ & $70(7.9 \%)$ & \\
\hline Assault & $14(1.6 \%)$ & $2(0.2 \%)$ & $0(0 \%)$ & $4(0.45 \%)$ & $20(2.2 \%)$ & \\
\hline Burn & $8(0.9 \%)$ & $0(0 \%)$ & $0(0 \%)$ & $2(0.2 \%)$ & $10(1.1 \%)$ & \\
\hline \multicolumn{7}{|l|}{ Disposition } \\
\hline Ward admission & $508(57.3 \%)$ & $32(3.6 \%)$ & $46(5.2 \%)$ & $32(3.6 \%)$ & $618(69.7 \%)$ & $<0.00001$ \\
\hline Discharge & $140(15.8 \%)$ & $0(0 \%)$ & $0(0 \%)$ & $0(0 \%)$ & $140(15.8 \%)$ & \\
\hline Leave against medical advice & $68(7.7 \%)$ & $0(0 \%)$ & $0(0 \%)$ & $0(0 \%)$ & $68(7.7 \%)$ & \\
\hline ICU admission & $0(0 \%)$ & $2(0.2 \%)$ & $8(0.9 \%)$ & $26(2.9 \%)$ & $36(4 \%)$ & \\
\hline Operation Theatre & $8(0.9 \%)$ & $2(0.2 \%)$ & $0(0 \%)$ & $12(1.35 \%)$ & $22(2.5 \%)$ & \\
\hline Death & $0(0 \%)$ & $0(0 \%)$ & $0(0 \%)$ & $2(0.2 \%)$ & $2(0.2 \%)$ & \\
\hline
\end{tabular}

ISS was calculated based on MDCALC calculator implanted in our CERNER (Information Technology based emergency department document system) 
Table 4. Trauma mechanisms in relation to RTS

\begin{tabular}{|c|c|c|c|c|}
\hline Variable & RTS $>5$ & RTS $\leq 5$ & Total & $P$ value \\
\hline Patients & $870(98.2 \%)$ & $16(1.8 \%)$ & 886 & \\
\hline \multicolumn{5}{|l|}{ Age } \\
\hline Average (y) & $31 \pm 14.16$ & $28 \pm 14.9$ & & \\
\hline \multicolumn{5}{|l|}{ Gender } \\
\hline Male & $704(79.46 \%)$ & $14(1.58 \%)$ & 718 (81.04\%) & \\
\hline Female & $166(18.73 \%)$ & $2(0.22 \%)$ & $168(18.96 \%)$ & \\
\hline \multicolumn{5}{|l|}{ Trauma type } \\
\hline Motor vehicle collision & $474(53.5 \%)$ & $8(0.9 \%)$ & $482(54.4 \%)$ & 0.0163 \\
\hline Pedestrian & $90(10 \%)$ & $0(0 \%)$ & $90(10 \%)$ & \\
\hline Fall & $94(10.6 \%)$ & $2(0.2 \%)$ & $190(21.4 \%)$ & \\
\hline Bicycle/motor bike & $66(7.7 \%)$ & $2(0.2 \%)$ & $70(7.9 \%)$ & \\
\hline Assault & $20(2.25 \%)$ & $2(0.2 \%)$ & $22(2.5 \%)$ & \\
\hline Burn & $10(1.1 \%)$ & $0(0 \%)$ & $10(1.1 \%)$ & \\
\hline Others & $20(2.25 \%)$ & $2(0.2 \%)$ & $22(2.5 \%)$ & \\
\hline \multicolumn{5}{|l|}{ Disposition } \\
\hline Ward admission & $616(69.5 \%)$ & $2(0.2 \%)$ & $618(69.7 \%)$ & $<0.00001$ \\
\hline Discharge & $140(15.8 \%)$ & $0(0 \%)$ & $140(15.8 \%)$ & \\
\hline Leave against medical advice & $68(7.7 \%)$ & $0(0 \%)$ & $68(7.7 \%)$ & \\
\hline ICU admission & $22(2.5 \%)$ & $14(1.5 \%)$ & $36(4 \%)$ & \\
\hline Operation Theatre & $22(2.5 \%)$ & $0(0 \%)$ & $22(2.5 \%)$ & \\
\hline Death & $0(0 \%)$ & $2(0.2 \%)$ & $2(0.2 \%)$ & \\
\hline
\end{tabular}

Table 5. Diurnal variation in regard to the shift time

\begin{tabular}{lc}
\hline Time of the day & Number of patients \\
\hline Morning (0700-1500) & $270(30.4 \%)$ \\
\hline Evening (1500-2300) & $460(51.9 \%)$ \\
\hline Night $(2300-0700)$ & $156(17.6 \%)$ \\
\hline Total & 886 \\
\hline
\end{tabular}

$P$ value $<0.00001$.

\section{Discussion}

The number of patients with positive imaging results was less than those who had normal tests with no injuries at all. This mainly happens due to the hospital policy in trauma code activation or less likely due to variable training among the emergency physicians, resulting in more imaging and unnecessary admissions, although we had not missed any life-threatening injuries. This finding should be weighed against the radiation exposure and cost effectiveness of trauma CT performance. Additionally, it should direct the attention of physicians to perform a detailed physical examination and pay extra attention to the mechanism of injury before activating a trauma code. The percentage of male patients continued to constitute the highest number of causalities and this was mainly due to the cultural nature of the city, and car accidents were the main cause of trauma in our hospital leading to a trauma code activation. This finding is in line with many worldwide statistics (8). Head and neck injuries accounted for the highest body parts injured and they were primarily related to the high-speed motor vehicle collisions and falls. This finding is consistent with other studies $(9,10)$. ISS was found to be more sensitive than RTS in detecting the severity of the injury; however, it is more difficult to calculate and imaging is needed. Also, there is a necessity for a system-based calculator for easier estimation (11). The diurnal variations in trauma clearly point out that rush time and working hours contributed to the majority of accidents. This result is corroborated with the findings obtained by Pape-Köhler et al at Private University of Witten/Herdecke in Germany (12). Thus, hospital staff should be more aware and prepared at these conditions with the availability of all specialties and consultants oncall for possibly better outcomes.

Pedestrian injuries although dangerous but were lower than falls, and this was mainly due to excellent road traffic regulations and strict rules in our city as compared to previous years (13); however, extra care should be directed towards falls, and extra safety regulations should be in place with strict supervision of already available ones.

\section{Limitations}

One limitation of this study is the paucity of previous research on the subject in our hospital. Thus, no groundwork thesis was done to compare with and see where we are now. Besides, we did not find similar studies in the neighbouring hospital or other trauma studies in other cities in U.A.E. It is worth mentioning that the results of this study cannot be applicable in other hospitals due to different hospital polices and different emergency physician training schools. 


\section{Conclusion}

Detailed physical examination and a more teamworkbased decision should be in place to activate a trauma code, before exposing patients to unnecessary high radiation. Moreover, hospital policy in activating a trauma code should be revised, with more care being paid to the mechanisms of injury and the condition of the patient, without missing any injury that could harm the patient and put the physicians under scrutiny and legal issues. The emergency room should be better prepared during peak times, with the availability of senior ED consultants to provide high-quality care to trauma patients, and decrease unneeded imaging.

\section{Ethical Issues}

None to be declared.

\section{Authors' contributions}

MME and MA contributed to the design and implementation of the research, analysis of the results as well as writing of the manuscript.

\section{References}

1. WHO. About the Global Burden of Disease (GBD) project [Internet]. Who; 2020. Available from: https:// www.who.int/healthinfo/global_burden_disease/ about/en/.

2. Feliciano, DV, Mattox K, Moore EE. Trauma. 6th ed. New York: McGraw-Hill; 2008.

3. Alleway R. NCEPOD - Trauma: Who Cares? Report (2007) [Internet]. Ncepod.org.uk. 2007 . Available from: https://www.ncepod.org.uk/2007t.html

4. Haagsma JA, Graetz N, Bolliger I, Naghavi M, Higashi $\mathrm{H}$, Mullany EC, et al. The global burden of injury: incidence, mortality, disability-adjusted life years and time trends from the Global Burden of Disease study 2013. Inj Prev 2016; 22(1): 3-18. doi: 10.1136/ injuryprev-2015-041616.

5. Palanca S, Taylor DM, Bailey M, Cameron PA. Mechanisms of motor vehicle accidents that predict major injury. Emerg Med (Fremantle) 2003; 15(5-6): 423-8. doi: 10.1046/j.1442-2026.2003.00496.x.
6. Haider AH, Chang DC, Haut ER, Cornwell EE 3rd, Efron DT. Mechanism of injury predicts patient mortality and impairment after blunt trauma. J Surg Res 2009; 153(1): 138-42. doi: 10.1016/j. jss.2008.04.011.

7. Lerner EB, Shah MN, Cushman JT, Swor RA, Guse $\mathrm{CE}$, Brasel K, et al. Does mechanism of injury predict trauma center need? Prehosp Emerg Care 2011; 15(4): 518-25. doi: 10.3109/10903127.2011.598617.

8. Yadollahi M, Ghiassee A, Anvar M, Ghaem H, Farahmand M. Analysis of Shahid Rajaee hospital administrative data on injuries resulting from car accidents in Shiraz, Iran: 2011-2014 data. Chin J Traumatol 2017; 20(1): 27-33. doi: 10.1016/j. cjtee.2015.10.006.

9. Heim C, Bosisio F, Roth A, Bloch J, Borens O, Daniel $\mathrm{RT}$, et al. Is trauma in Switzerland any different? epidemiology and patterns of injury in major trauma - a 5-year review from a Swiss trauma centre. Swiss Med Wkly 2014; 144: w13958. doi: 10.4414/ smw.2014.13958.

10. Bolandparvaz S, Yadollahi M, Abbasi HR, Anvar M. Injury patterns among various age and gender groups of trauma patients in southern Iran: a cross-sectional study. Medicine (Baltimore) 2017; 96(41): e7812. doi: 10.1097/md.0000000000007812.

11. Javali RH, Krishnamoorthy, Patil A, Srinivasarangan M, Suraj, Sriharsha. Comparison of injury severity score, new injury severity score, revised trauma score and trauma and injury severity score for mortality prediction in elderly trauma patients. Indian J Crit Care Med 2019; 23(2): 73-7. doi: 10.5005/jpjournals-10071-23120.

12. Pape-Köhler CI, Simanski C, Nienaber U, Lefering R. External factors and the incidence of severe trauma: time, date, season and moon. Injury 2014; 45 Suppl 3: S93-9. doi: 10.1016/j.injury.2014.08.027.

13. Hefny AF, Eid HO, Abu-Zidan FM. Pedestrian injuries in the United Arab Emirates. Int J Inj Contr Saf Promot 2015; 22(3): 203-8. doi: $10.1080 / 17457300.2014 .884143$. 